Journal of Mathematics and Statistics 3 (3) : 100-105, 2007

ISSN 1549-3644

(C) 2007 Science Publications

\title{
Study of Families of Curves in the Euclidian Plan
}

\author{
${ }^{1}$ Belaib Lekhmissi, ${ }^{2}$ Bouarroudj Nadra and ${ }^{3}$ Hocine El Habib \\ ${ }^{1}$ Department of Mathematics, Faculty of Sciences, University d'Oran, Es-Sénia Oran Algeria \\ ${ }^{2}$ Department of Mathematics and Informatics, E.N.S.E.T Oran, Algeria \\ ${ }^{3}$ Department of Informatics, Oran's University, Algeria
}

\begin{abstract}
Non-standard analysis techniques are more considered in approaching complex mathematical domains. By using some concepts of non-standard analysis methods such as regionalization method, we deal with a family of curves in an Euclidian plan. The solutions of the algebraic equations representing these curves in a plan have an hyperbolic forms.
\end{abstract}

Key words: Non-standard analysis, regionalization, unlimited number, infinitesimal, appreciable

\section{INTRODUCTION}

Our recent work deals with a family of curves in the Euclidian plan by using some concepts of nonstandard analysis given by Robinson, $\mathrm{A}^{[1]}$ and axiomatized by Nelson, E. ${ }^{[2]}$. More precisely, under some conditions concerning domains we show that the solutions of the algebraic curves have geometrical forms (hyperbolic).

we start our study with the algebraic curve

$E(m, n, a)$ defined in $\mathfrak{R}_{+}^{* 2}$ by the set

$E(m, n, a)=\left\{(x, y) \in \Re_{+}^{2} /\left(\frac{1}{x}\right)^{2 m}+\left(\frac{1}{y}\right)^{2 n}=a, \mathrm{~m} \geq \mathrm{n} \succ 0, \mathrm{a}>0\right\}$

where $(x, y)$ verify the following equation $x^{2 m} y^{2 n} a=y^{2 n}+x^{2 m}, \mathrm{a}>0$ real $\mathrm{x}>0 \quad, \mathrm{y}>0$ by using the regionalization method ${ }^{[3]}$.

This curve allows us to define two sets $Q\left(a^{-\frac{1}{2 m}}, a^{-\frac{1}{2 n}}\right)$ and $Q\left(\left(\frac{a}{2}\right)^{-\frac{1}{2 m}},\left(\frac{a}{2}\right)^{-\frac{1}{2 n}}\right)$ such that $Q\left(a^{-\frac{1}{2 m}}, a^{-\frac{1}{2 n}}\right) \quad$ the quadrant defined by $x \geq a^{-\frac{1}{2 m}}$ and $y \geq a^{-\frac{1}{2 n}}$ and the vertex $\left(a^{-\frac{1}{2 m}}, a^{-\frac{1}{2 n}}\right)$ and $Q\left(\left(\frac{a}{2}\right)^{-\frac{1}{2 m}},\left(\frac{a}{2}\right)^{-\frac{1}{2 n}}\right)$ the quadrant defined by $\quad x \geq\left(\frac{a}{2}\right)^{-\frac{1}{2 m}}, y \geq\left(\frac{a}{2}\right)^{-\frac{1}{2 n}}$ and the vertex $\left(\left(\frac{a}{2}\right)^{-\frac{1}{2 m}},\left(\frac{a}{2}\right)^{-\frac{1}{2 n}}\right)$; which allow us to cover the curve $E(m, n, a)$.

From the equation $\left(\frac{1}{x}\right)^{2 m}+\left(\frac{1}{y}\right)^{2 n}=a$ which defines the curve $E(m, n, a)$ and we can write the function

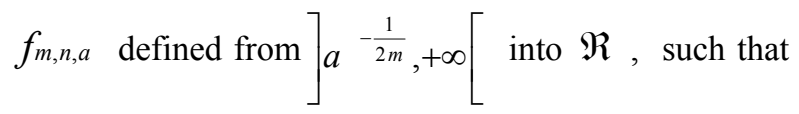
$f_{m, n, a}(x)=\frac{x^{\frac{m}{n}}}{\left(a x^{2 m}-1\right)^{\frac{1}{2 n}}}$

Proposition 1: The function $f_{m, n, a}$ has the following properties:

$\left.1^{\circ}\right) f_{m, n, a}(x)$ is strictly decreasing on $] a^{-\frac{1}{2 m}},+\infty[$. $\left.2^{\circ}\right) f_{m, n, a}(x)$ has $y=a^{-\frac{1}{2 n}}$ as horizontal asymptote $\left.3^{\circ}\right) f_{m, n, a}(x)$ has $x=a^{-\frac{1}{2 m}}$ as vertical asymptote

Proof of the proposition 1: We show that $f_{m, n, a}(x)$ is strictly decreasing; we study the sign of its derivable form:

Corresponding Author: Belaib Lekhmissi, Department of Mathematics, Faculty of Sciences, University d'Oran, EsSénia,Oran Algeria 
Given: $\quad f_{m, n, a}^{\prime}(x)=\frac{-\frac{m}{n} x^{\frac{m}{n}}}{x\left(a x^{2 m}-1\right)^{\frac{1}{2 n}+1}}$

Since $x$ belongs $] a^{-\frac{1}{2 m}},+\infty[$ is equivalent to $x \succ a^{-\frac{1}{2 m}}$ equivalent $x^{2 m} \succ \frac{1}{a}$

Since $-\frac{m}{n}<0$ then $\frac{-\frac{m}{n} x^{\frac{m}{n}}}{x\left(a x^{2 m}-1\right)^{\frac{1}{k^{n}}+1}}<0$

However $x\left(a x^{2 m}-1\right)^{\frac{1}{2 n+1}}>0$ then

$\frac{x^{\frac{m}{n}}}{x\left(a x^{2 m}-1\right)^{\frac{1}{2^{2}+1}}}>0$ where $f^{\prime} \prec 0$ then the function $f$

is decreasing.

2- We are going to verify that $f$ has an horizontal asymptote, for this we compute

$$
\begin{aligned}
& \lim _{x \rightarrow \infty} f_{m, n, a}(x)=\lim _{x \rightarrow \infty} \frac{x^{\frac{m}{n}}}{\left(a x^{2 m}-1\right)^{\frac{1}{2 n}}} \\
& =\lim _{x \rightarrow \infty} \frac{x^{\frac{m}{n}}}{a^{\frac{1}{2 n}} x^{\frac{m}{n}}}=a^{-\frac{1}{2 n}}
\end{aligned}
$$

Then $y=a^{-\frac{1}{2 n}}$ is an horizontal asymptote.

3- We are going to verify that $f$ has a vertical asymptote for this we compute

$$
\lim _{x \rightarrow a^{-\frac{1}{2 m}}} f_{m, n, a}(x)=\lim _{x \rightarrow a^{-\frac{1}{2 m}}} \frac{x^{\frac{m}{n}}}{\left(a x^{2 m}-1\right)^{\frac{1}{2 n}}}=+\infty
$$

Then $x=a^{-\frac{1}{2 m}}$ is a vertical asymptote.

Lemma d'encadrement : We have the relations : $\left(\left(\frac{a}{2}\right)^{-\frac{1}{2 m}},\left(\frac{a}{2}\right)^{-\frac{1}{2 n}}\right) \in E(m, n, a) \subset Q\left(a^{-\frac{1}{2 m}}, a^{-\frac{1}{2 n}}\right)-Q^{0}\left(\left(\frac{a}{2}\right)^{-\frac{1}{2 m}},\left(\frac{a}{2}\right)^{-\frac{1}{2 n}}\right)$ with $\mathrm{Q}^{\mathrm{o}}$ the interior part of $\mathrm{Q}$.

Proof of the lemma d'encadrement: The vertex $\mathcal{Q}\left(\left(\frac{a}{2}\right)^{-\frac{1}{2 m}},\left(\frac{a}{2}\right)^{-\frac{1}{2 n}}\right)$ belongs to $E(m, n, a)$

$$
\begin{aligned}
& \text { because }\left[\frac{1}{\left(\frac{a}{2}\right)^{-\frac{1}{2 m}}}\right]^{2 m}+\left[\frac{1}{\left(\frac{a}{2}\right)^{-\frac{1}{2 n}}}\right]^{2 n}=\frac{a}{2}+\frac{a}{2}=a \\
& \left(\left(\frac{a}{2}\right)^{-\frac{1}{2 m}},\left(\frac{a}{2}\right)^{-\frac{1}{2 n}}\right) \in E(m, n, a) \\
& \left(x_{0}, y_{0}\right) \in Q^{0}\left[a^{-\frac{1}{2 m}}, a^{-\frac{1}{2 n}}\right] \subset Q\left[a^{-\frac{1}{2 m}}, a^{-\frac{1}{2 n}}\right],
\end{aligned}
$$

following the definition of an interior of a set.

(ii) we show that $E(m, n, a) \Rightarrow\left(\frac{1}{x_{0}}\right)^{2 m}+\left(\frac{1}{y_{0}}\right)^{2 n}=a$

It remains to be shown that

$$
\begin{aligned}
& \left(x_{0}, y_{0}\right) \notin Q^{0}\left(\left(\frac{a}{2}\right)^{-\frac{1}{2 m}},\left(\frac{a}{2}\right)^{-\frac{1}{2 n}}\right) \\
& \text { If } \left.\quad\left(x_{0}, y_{0}\right) \notin Q \int\left(\frac{a}{2}\right)^{-\frac{1}{2 m}},\left(\frac{a}{2}\right)^{-\frac{1}{2 n}}\right) \\
& \left(x_{0}, y_{0}\right) \notin Q^{0}\left(\left(\frac{a}{2}\right)^{-\frac{1}{2 m}},\left(\frac{a}{2}\right)^{-\frac{1}{2 n}}\right)
\end{aligned}
$$

then

By contradiction we suppose that $\left(x_{0}, y_{0}\right) \in Q^{0} \subset Q\left(\left(\frac{a}{2}\right)^{-\frac{1}{2 m}},\left(\frac{a}{2}\right)^{-\frac{1}{2 n}}\right)-\left\{\left(\frac{a}{2}\right)^{-\frac{1}{2 m}},\left(\frac{a}{2}\right)^{-\frac{1}{2 n}}\right\}$

If we take a point $\left(x_{0}, y_{0}\right) \in Q^{0} \subset Q\left(\left(\frac{a}{2}\right)^{-\frac{1}{2 m}},\left(\frac{a}{2}\right)^{-\frac{1}{2 n}}\right)-\left\{\left(\frac{a}{2}\right)^{-\frac{1}{2 m}},\left(\frac{a}{2}\right)^{-\frac{1}{2 n}}\right\}$

Then

$\left.x_{0}\right\rangle\left(\frac{a}{2}\right)^{-\frac{1}{2 m}}$ and $y_{0} \geq\left(\frac{a}{2}\right)^{-\frac{1}{2 n}}$ or $x_{0} \geq\left(\frac{a}{2}\right)^{-\frac{1}{2 m}}$ and $\left.y_{0}\right\rangle\left(\frac{a}{2}\right)^{-\frac{1}{2 n}}$

Imply $\left(x_{0}, y_{0}\right) \notin E(m, n, a)$, hence contradiction. 
Lemma of general framing: We have the following relations:

$\left(\left(\frac{a}{k}\right)^{-\frac{1}{2 m}},\left(\frac{a(k-1)}{k}\right)^{-\frac{1}{2 n}}\right) \in E(m, n, a) \subset Q\left[a^{-\frac{1}{2 m}}, a^{-\frac{1}{2 n}}\right]-Q^{0}\left[\left(\frac{a}{k}\right)^{-\frac{1}{2 m}},\left(\frac{a(k-1)}{k}\right)^{-\frac{1}{2 n}}\right]$

\section{Proof of the lemma of general framing:}

Let $\left(x_{0}, y_{0}\right) \in E(m, n, a) \Rightarrow\left(\frac{1}{x_{0}}\right)^{2 m}+\left(\frac{1}{y_{0}}\right)^{2 n}=a$

$\left(\frac{1}{x_{0}}\right)^{2 m}\left\langle a\right.$ and $\left(\frac{1}{y_{0}}\right)^{2 n}\left\langle a\right.$ then $\left.x_{0}\right\rangle a^{-\frac{1}{2 m}}$ and $\left.y_{0}\right\rangle a^{-\frac{1}{2 n}}$

Imply $\left(x_{0}, y_{0}\right) \in Q^{0}\left(a^{-\frac{1}{2 m}}, a^{-\frac{1}{2 n}}\right) \subset Q\left(a^{-\frac{1}{2 m}}, a^{-\frac{1}{2 n}}\right)$

It remains to be shown that $\left(x_{0}, y_{0}\right) \notin Q^{0}\left[\left(\frac{a}{k}\right)^{-\frac{1}{2 m}},\left(\frac{a(k-1)}{k}\right)^{-\frac{1}{2 n}}\right]$

By contradiction we suppose

$\left(x_{0}, y_{0}\right) \in Q^{0} \subset Q\left[\left(\frac{a}{k}\right)^{-\frac{1}{2 m}},\left(\frac{a(k-1)}{k}\right)^{-\frac{1}{2 n}}\right]-\left\{\left(\frac{a}{k}\right)^{-\frac{1}{2 m}},\left(\frac{a(k-1)}{k}\right)^{-\frac{1}{2 n}}\right\}$.

if we take a point

$\left(x_{0}, y_{0}\right) \in Q^{0}\left[\left(\frac{a}{k}\right)^{-\frac{1}{2 m}},\left(\frac{a(k-1)}{k}\right)^{-\frac{1}{2 n}}\right]-\left\{\left(\left(\frac{a}{k}\right)^{-\frac{1}{2 m}},\left(\frac{a(k-1)}{k}\right)^{-\frac{1}{2 n}}\right)\right\}$

$\left.x_{0}\right\rangle\left(\frac{a}{k}\right)^{-\frac{1}{2 m}} \quad$ and $\quad y_{0} \geq\left(\frac{a(k-1)}{k}\right)^{-\frac{1}{2 n}}$

then $\left(x_{0}, y_{0}\right) \notin E(m, n, a)$

where $\quad x_{0} \geq\left(\frac{a}{k}\right)^{-\frac{1}{2 m}}$ and $y_{0}>\left(\frac{a(k-1)}{k}\right)^{-\frac{1}{2 n}}$

Contradiction

Proposition 2: When $m \geq n>0$ are integers the geometric place of the vertex of the quadrants

$Q\left(a^{-\frac{1}{2 m}}, a^{-\frac{1}{2 n}}\right)$ and $Q\left[\left(\frac{a}{2}\right)^{-\frac{1}{2 m}},\left(\frac{a}{2}\right)^{-\frac{1}{2 n}}\right]$ is the

curve of equation $y=x^{\frac{m}{n}}$
Proof of the proposition 2: Since the vertex of the quadrants

$Q\left(a^{-\frac{1}{2 m}}, a^{-\frac{1}{2 n}}\right) \quad$ and $Q\left(\left(\frac{a}{2}\right)^{-\frac{1}{2 m}},\left(\frac{a}{2}\right)^{-\frac{1}{2 n}}\right)$

are $\left(a^{-\frac{1}{2 m}}, a^{-\frac{1}{2 n}}\right) \quad$ and $\left(\left(\frac{a}{2}\right)^{-\frac{1}{2 m}},\left(\frac{a}{2}\right)^{-\frac{1}{2 n}}\right)$

the writing $a^{-\frac{1}{2 n}}=\left(a^{\frac{1}{2 m}}\right)^{\frac{m}{n}} \quad$ and $\left(\frac{a}{2}\right)^{\frac{1}{2 n}}=\left(\left(\frac{a}{2}\right)^{\frac{1}{2 m}}\right)^{\frac{m}{n}}$

shows that the vertex verify the equation $\quad \mathrm{y}=\mathrm{x}^{\frac{m}{n}}$.

Proposition 3: When $m \geq n>0$ are fixed integers and a fixed real $a=2 x_{0}^{-2 m}=2 y_{0}^{-2 n}$ the geometric place of the vertex of the quadrants

$Q\left(a^{-\frac{1}{2 m}}, a^{-\frac{1}{2 n}}\right)$ and $\left(\operatorname{resp} Q\left(\left(\frac{a}{k}\right)^{-\frac{1}{2 m}},\left(\frac{a(k-1)}{k}\right)^{-\frac{1}{2 n}}\right)\right.$

is the curve of equation $y=x^{\frac{m}{n}} \quad($ resp $\left.y=(k-1)^{-\frac{1}{2 n}} x^{\frac{m}{n}}\right)$ when $a$ ranges over $] 0,+\infty[$

Proof of the proposition 3: The vertex of the quadrants

$Q\left(a^{-\frac{1}{2 m}}, a^{-\frac{1}{2 n}}\right) \quad$ and $Q\left(\left(\frac{a}{k}\right)^{-\frac{1}{2 m}},\left(\frac{a(k-1)}{k}\right)^{-\frac{1}{2 n}}\right)$

are

$S\left(a^{-\frac{1}{2 m}}, a^{-\frac{1}{2 n}}\right) \quad$ and $S\left(\left(\frac{a}{k}\right)^{-\frac{1}{2 m}},\left(\frac{a(k-1)}{k}\right)^{-\frac{1}{2 n}}\right)$.

The writing $a^{-\frac{1}{2 n}}=\left(a^{-\frac{1}{2 m}}\right)^{\frac{m}{n}}$ shows that the coordinates of $S\left(a^{-\frac{1}{2 m}}, a^{-\frac{1}{2 n}}\right)$. Verify the equation $\mathrm{y}=\mathrm{x}^{\frac{m}{n}}$. 
The writing $\left(\frac{a(k-1)}{k}\right)^{-\frac{1}{2 n}}=(k-1)^{-\frac{1}{2 n}}\left(\left(\frac{a}{k}\right)^{-\frac{1}{2 m}}\right)^{\frac{m}{n}}$ shows that the coordinates of $S\left(\left(\frac{a}{k}\right)^{-\frac{1}{2 m}},\left(\frac{a(k-1)}{k}\right)^{-\frac{1}{2 n}}\right)$ Verify the equation $y=(k-1)^{-\frac{1}{2 n}} x^{\frac{m}{n}}$

Reciprocaly: A point $\left(x_{0}, y_{0}\right)$ of the curve $y=(k-1)^{-\frac{1}{2 n}} x^{\frac{m}{n}}$ is the vertex of the quadrant $Q\left(\left(\frac{a}{k}\right)^{-\frac{1}{2 m}},\left(\frac{a(k-1)}{k}\right)^{-\frac{1}{2 n}}\right)=Q\left(\left(x_{0}^{-2 m}\right)^{\frac{1}{2 m}},\left(y_{0}^{-2 n}\right)^{\frac{1}{2 n}}\right)$. $\left.Q\left(k \frac{x_{0}^{-2 m}}{k}\right)^{\frac{1}{2 m}},\left(\frac{(k-1)}{k} \cdot \frac{k}{(k-1)} y_{0}^{-2 n}\right)^{\frac{1}{2 n}}\right)=Q\left(\left(\frac{a}{k}\right)^{\frac{1}{2 m}},\left(\frac{d(k-1)}{k}\right)^{\frac{1}{2 n}}\right)$

From the equality

$k \frac{x_{0}^{-2 m}}{k}=\frac{a}{k}$ we obtain $a=k \cdot x_{0}^{-2 m}$

From the $\frac{(k-1)}{k} \cdot \frac{k}{(k-1)} y_{0}^{-2 n}=\frac{k-1}{k}$ a weobtaina $=\frac{k}{k-1} y_{0}^{-2 n}$ which give us: $a=k \cdot x_{0}^{-2 m}=\frac{k}{k-1} y_{0}^{-2 n} \quad ;$ hence the point $\left(x_{0}, y_{0}\right)$ is a curve point.

Parameters monitoring the shape of the curves:

Let $\quad V=\left(\frac{a}{2}\right)^{-\frac{1}{2 n}}-a^{-\frac{1}{2 n}}=\left(1-2^{-\frac{1}{2 n}}\right)\left(\frac{a}{2}\right)^{-\frac{1}{2 n}}$

Vertical thickness of the « main band of encadrement ».

Let $\quad h=\left(\frac{a}{2}\right)^{-\frac{1}{2 m}}-a^{-\frac{1}{2 m}}=\left(1-2^{-\frac{1}{2 m}}\right)\left(\frac{a}{2}\right)^{-\frac{1}{2 m}}$

Horizontal thickness of the «main band of encadrement $»$.
Let $\quad r=\frac{m}{n}$ Parameter monitoring the curve $C(m, n)$ of the vertex of the quadrants.

$Q\left(a^{-\frac{1}{2 m}}, a^{-\frac{1}{2 n}}\right)$ and $Q\left(\left(\frac{a}{2}\right)^{-\frac{1}{2 m}},\left(\frac{a}{2}\right)^{-\frac{1}{2 n}}\right)$.

And let $C(m, n):$ The curve of equation $y=x^{\frac{m}{n}}$

Comparaison of the thickness: We have two situations

(i) $\frac{m}{n} \approx 1$ the $C(m, n)$ curve has the shape of the right-line $y=x$

(ii) $1 \prec \prec \frac{m}{n} \prec \prec \infty$ the $C(m, n)$ curve has the shape of the right-line $y=x^{\left(\frac{m}{n}\right)^{o}}$

Proposition 4: If $n \succ 0$ is infinitely big, then the vertical thickness $\mathrm{V}$ is substantially positive if and only if : $a \in\left(\frac{A_{+}}{2 n}\right)^{2 n}$

Proof of proposition 4: We show that $V=\left(1-2^{-\frac{1}{2 n}}\right)\left(\frac{a}{2}\right)^{-\frac{1}{2 n}} \in A_{+}$is equivalent to $a \in\left(\frac{A_{+}}{2 n}\right)^{2 n}$ Indeed:

$\left(1-2^{-\frac{1}{2 n}}\right)\left(\frac{a}{2}\right)^{-\frac{1}{2 n}} \in A_{+}$is equivalent to $\left(\frac{a}{2}\right)^{-\frac{1}{2 n}} \in\left(\frac{A_{+}}{1-2^{-\frac{1}{2 n}}}\right)$

We apply the limited development of $2^{-\frac{1}{2 n}}=e^{-\frac{1}{2 n} \log 2}$

$2^{-\frac{1}{2 n}}=e^{-\frac{1}{2 n} \log 2}=$

$=1-\frac{1}{2 n} \log 2+\frac{1}{2 !}\left(\frac{1}{2 n} \log 2\right)^{2}-\frac{1}{3 !}\left(\frac{1}{2 n} \log 2\right)^{3}+\ldots$.

$=1-\frac{1}{2 n} \log 2\left[1-\frac{1}{2 !}\left(\frac{1}{2 n} \log 2\right)+\frac{1}{3 !}\left(\frac{1}{2 n} \log 2\right)^{2}+\ldots ..\right]$

we take $\gamma=1-\frac{1}{2 !}\left(\frac{1}{2 n} \log 2\right)+\frac{1}{3 !}\left(\frac{1}{2 n} \log 2\right)^{2}+\ldots$ 
$\gamma \approx 1 \quad$ If $\mathrm{n}>0$ is infinitely big, then $2^{-\frac{1}{2 n}}=1-\left(\frac{1}{2 n} \log 2\right) \gamma \approx 1-\frac{1}{2 n} \log 2$

Therefore $\left(\frac{a}{2}\right)^{-\frac{1}{2 n}} \in \frac{A_{+}}{1-1+\frac{1}{2 n} \log 2}=\frac{A_{+}}{\frac{1}{2 n} \log 2}$

$\frac{a}{2} \in\left(\frac{A_{+}}{\frac{1}{2 n} \log 2}\right)^{-2 n}$

isequivaletwot $\frac{a}{2} \in\left(\frac{2 n \cdot A_{+}}{\log 2}\right)^{-2 n}$ isequivalethot $\frac{a}{2} \in\left(\frac{\log }{2 n A}\right)^{2 n}$

As $\frac{\log 2}{A_{+}} \approx A_{+}$therefore

Thus $\left(\frac{P}{2 n}\right)^{2 n},\left(\frac{G_{+}}{2 n}\right)^{2 n} \quad \begin{aligned} & a \in\left(\frac{A_{+}}{2 n}\right)^{2 n} \text { c.q.e.d } \\ & \text { Are the complemen }\end{aligned}$ $\left(\frac{A_{+}}{2 n}\right)^{2 n}$

The graphical representation:

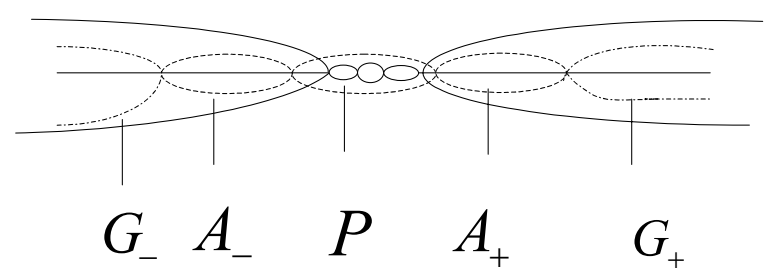

Proposition 5: If $m \succ 0$ is infinitely big, then the horizontal thickness $\mathrm{h}$ is substantial positive if and only if $a \in\left(\frac{A_{+}}{2 n}\right)$

Proof of proposition 5: The same proof as for the proposition 4 by substituting $m$ for $n$.

Proposition 6: If $m=n \delta, \delta \in 1+\frac{L}{\ln 2 n}$ then we have $\left(\frac{A_{+}}{2 m}\right)^{2 m}=\left(\frac{A_{+}}{2 n}\right)^{2 n}$

with $\mathrm{L}$ : limit, $\mathrm{A}_{+}$: substantial positive.
Study of the tangents: Let $E(m, n, a)$ a family of curves of the plan $\mathfrak{R}^{2}$ defined by the equation $\left(\frac{1}{x}\right)^{2 m}+\left(\frac{1}{y}\right)^{2 n}=a$

( $m \geq n \geq 0$ ) infinitely big integer, a $>0$ real. the tangent in point $\left(x_{0}, y_{0}\right)$ of the $E(m, n, a)$ curve has the equation $n\left(y-y_{0}\right) x_{0}{ }^{2 m+1}+m\left(x-x_{0}\right) y_{0}{ }^{2 n+1}=0 . \quad$ Indeed: the equation of the tangent in a point $\left(x_{0}, y_{0}\right)$ is:

$y-y_{0}=f_{m, n, a}^{\prime}\left(x_{0}\right)\left(x-x_{0}\right)$

Where $y=f_{m, n, a}(x)=\frac{x^{\frac{m}{n}}}{\left(a x^{2 m}-1\right)^{\frac{1}{2 n}}}$

then $f_{m, n, a}^{\prime}(x)=\frac{-\frac{m}{n} x^{\frac{m}{n}-1}}{\left(a x^{2 m}-1\right)^{\frac{1}{2 n}+1}}$

And since $y^{2 n}=\frac{x^{2 m}}{a x^{2 m}-1}$, we replace in $\left(^{*}\right)$ we obtain $y-y_{0}=\frac{-\frac{m}{n} x_{0}{ }^{\frac{m}{n}-1}}{\left(a x_{0}{ }^{2 m}-1\right)^{\frac{1}{2 n}+1}}\left(x-x_{0}\right)$

hence

$$
\begin{aligned}
& \left(y-y_{0}\right)\left(a x_{0}{ }^{2 m}-1\right)=\frac{-\frac{m}{n} x_{0}{ }^{\frac{m}{n}}}{x_{0}\left(a x_{0}{ }^{2 m}-1\right)^{\frac{1}{2 n}}}\left(x-x_{0}\right) \\
& \left(y-y_{0}\right) \frac{x_{0}{ }^{2 m}}{y_{0}{ }^{2 n}}=-\frac{m}{n} \frac{y_{0}}{x_{0}}\left(x-x_{0}\right)
\end{aligned}
$$

imply $\left(y-y_{0}\right) x_{0}{ }^{2 m+1}=-\frac{m}{n} y_{0}{ }^{2 n+1}\left(x-x_{0}\right)$

$n\left(y-y_{0}\right) x_{0}{ }^{2 m+1}+m y_{0}^{2 n+1}\left(x-x_{0}\right)=0$

as $\quad x_{0}>0, y_{0}>0$ we have the equation:

$y-y_{0}+\frac{m}{n} \frac{y_{0}^{2 n+1}}{x_{0}^{2 m+1}}\left(x-x_{0}\right)=0$ 
Situation where the slope is infinitely small:

$\frac{m}{n} \frac{y_{0}{ }^{2 n+1}}{x_{0}{ }^{2 m+1}} \in P$ is equivalent $y_{0}^{2 n+1} \in \frac{n}{m} x_{0}^{2 m+1} P$

is equivalent $y_{0} \in\left(\frac{n}{m} P\right)^{\frac{1}{2 n+1}} x_{0}^{\frac{2 m+1}{2 n+1}}$

Result 1: the slope is infinitely small $\left(x_{0}, y_{0}\right) \in \mathfrak{R}_{+}^{*_{2}}$

as $y_{0} \in\left(\frac{n}{m} P\right)^{\frac{1}{2 n+1}} x_{0}^{\frac{2 m+1}{2 n+1}}$

Situation where the slope is substantial positive: $\frac{m}{n} \frac{y_{0}{ }^{2 n+1}}{x_{0}{ }^{2 m+1}} \in A_{+}$is equivalent $y_{0} \in\left(\frac{n}{m} A_{+}\right)^{\frac{1}{2 n+1}} x_{0}^{\frac{2 m+1}{2 n+1}}$

if the slope is appreciable positive.

Result 2: the slope is substantial positive $(x, y) \in \mathfrak{R}_{+}^{* 2}$ as $y_{0} \in\left(\frac{n}{m} A_{+}\right)^{\frac{1}{2 n+1}} x_{0}^{\frac{2 m+1}{2 n+1}}$

Situation where the slope is infinitely big positive:

$\frac{m}{n} \frac{y_{0}{ }^{2 n+1}}{x_{0}{ }^{2 m+1}} \in G_{+}$is equivalent $y_{0} \in\left(\frac{n}{m} G_{+}\right)^{\frac{1}{2 n+1}} x_{0}^{\frac{2 m+1}{2 n+1}}$ if the

slope is infinitely great then :

Result 3: the slope is substantial positive as if $(x, y) \in \mathfrak{R}_{+}^{* 2}$ as $y_{0} \in\left(\frac{n}{m} G_{+}\right)^{\frac{1}{2 n+1}} x_{0}^{\frac{2 m+1}{2 n+1}}$
Parameters monitoring the shape of the curves (general case): In the general case $V_{k}$ and $h_{k}$ are equal:

$V_{k}=\left(1-\left(\frac{k-1}{k}\right)^{-\frac{1}{2 n}}\right)\left(\frac{a k}{k-1}\right)^{-\frac{1}{2 n}}$

thickness.

$k>1 \quad$ Vertical

$h_{k}=\left(1-k^{-\frac{1}{2 m}}\right)\left(\frac{a}{k}\right)^{-\frac{1}{2 m}}$

thickness bands

$\mathrm{k}>1 \quad$ horizontal

The curve $C_{k}(m, n)$ correspond to $E(m, n, a)$.

\section{CONCLUSION}

In this study we have introduced a non-standard analysis technique and regionalization for resolving algebraic curves formalized by algebraic equations

\section{REFERENCES}

1. Robinson, A., 1966. Non-Standard analysis. NorthHolland.

2. Nelson, E. 1977. Interior set theory, Bull Am. Math. Soc. 83: 1165- 1198.

3. Bobo Seke, 1988. Méthodes de régionalisations, quelques applications I.R.M.A, Strasbourg. 